Original Research Paper

\title{
Explicit Direct Integrators of RK Type for Solving Special Fifth-Order Ordinary Differential Equations
}

\author{
Mohammed S. Mechee and Murtaza A. Kadhim \\ Department of Mathematics, Faculty of Computer Science and Mathematics, Kufa University, Najaf, Iraq
}

Article history

Received: 26-02-2016

Revised: 23-12-2016

Accepted: 24-12-2016

Corresponding Author: Mohammed S. Mechee Department of Mathematics, Faculty of Computer Science and Mathematics, Kufa University, Najaf, Iraq

Email: mohammeds.abed@uokufa.edu.iq

\begin{abstract}
The main contribution of this paper is the development of direct explicit methods of Runge-Kutta (RK) type for solving special fifth-Order Ordinary Differential Equations (ODEs). For this purpose, we have generalized RKD and RKT methods of special third and fourth-order ODEs. Using Taylor expansion, we have derived the algebraic equations of algebraic equations of order conditions for the proposed RKM integrators up to the eighth order. Based on these conditions, two RKM methods of orders five and six with three and four-stage are derived. Numerical implementation shows that the new methods agree well with existing RK methods, but requires less function evaluations. This is so due to the fact that RKM methods are direct; hence, they save considerable amount of computational time.
\end{abstract}

Keywords: RK, RKN, RKD, RKT and RKFD Integrators, Runge-Kutta Methods, Special Fifth-Order, Four-Stage, Ordinary Differential Equations, Order Conditions, Taylor Series, Rooted Trees

\section{Introduction}

The mathematical modeling of many real-life problems in physics, engineering and economics can be written as higher-order differential equations, ordinary or partial, (DEs) model. Typical examples can be found in different fields such as quantum physics, solid state physics and fluid physics plasma physics Wazwaz (2006). For example, the nonlinear Korteweg-deVries $(\mathrm{KdV})$, the nonlinear Schrodinger and the KadomtsevPetviashvili equations are important mathematical models in quantum mechanics and nonlinear optics. Fifth-order KdV equation has found applications in some branches of physics, such as capillary-gravity water waves, chains of coupled oscillators and magnetoacoustic waves in plasma Isaza et al. (2015; Lee, 2014; Wazwaz, 2006). Another application of fifth-order DEs is the nonlinear dispersive equations, which include several models arising in the study of different physical phenomena Isaza et al. (2015). A mathematical model describing weakly nonlinear long internal waves at the interface between two thin layers of different density, called the Gardner-Kawahara equation, which is a generalization of the famous $\mathrm{KdV}$ equation, contains a cubic term together a fifth-order dispersion term Lee (2014).

In this study, a derivation for nonlinear equations (order conditions) of direct explicit RKM methods for solving a special fifth-order ODEs is presented, along with two numerical methods for ODEs with orders five and six.

\section{Special Fifth-Order Ordinary Differential Equations}

In this study, we concerned with fifth-order ordinary differential equation with no appearance for the first, second, third and fourth derivatives $w^{(i)}(x)$, for $i=$ $1,2,3,4$. It can be written in the following form:

$w^{(5)}(x)=g(x, w(x)), x \geq x_{0}$

Subject to initial condition:

$$
w^{(i)}\left(x_{0}\right)=\gamma^{i}
$$

for $i=0,1, \ldots, 4$.

where:

$$
g: \mathbb{R} \times \mathbb{R}^{N} \rightarrow \mathbb{R}^{N}
$$

and:

$$
w(x)=\left[w_{1}(x), w_{2}(x), \ldots, w_{N}(x)\right]
$$

for $i=0,1, \ldots, 4$. 
Knowing that, $N$ is the components number of the vector of independent variables of the system of ordinary differential Equation 1. To convert the function $g(x$, $w(x)$ ) which depends on two variables, to a function which depends only on one variable $w(x)$, using high dimension we can work in $N+1$ dimension using the assumption $w_{N+1}(x)=x$, then (1) can be simplified to following Equation 2:

$v^{(5)}(x)=h(v(x))$

Using the following consideration:

$$
v(x)=\left(\begin{array}{c}
w_{1}(x) \\
w_{2}(x) \\
w_{3}(x) \\
\ldots \\
\ldots \\
\ldots \\
w_{N}(x) \\
x
\end{array}\right), h(v)=\left(\begin{array}{c}
g_{1}\left(v_{1}, v_{2}, \ldots, v_{N}, v_{N+1}\right) \\
g_{2}\left(v_{1}, v_{2}, \ldots, v_{N}, v_{N+1}\right) \\
g_{3}\left(v_{1}, v_{2}, \ldots, v_{N}, v_{N+1}\right) \\
\ldots \\
\ldots \\
\ldots \\
g_{N}\left(v_{1}, v_{2}, \ldots, v_{N}, v_{N+1}\right) \\
0
\end{array}\right)
$$

Subject to the initial condition:

$$
w^{(i)}\left(x_{0}\right)=\overline{\gamma^{i}}
$$

for $i=0,1, \ldots, 4$.

where:

$$
\overline{\gamma^{i}}=\left[\gamma_{1}^{i}, \gamma_{2}^{i}, \ldots, \gamma_{N}^{i}, x_{0}\right]
$$

for $i=0,1, \ldots, 4$.

These class of ODEs are found in many engineering and physical problems. Some of scientists and engineers can solve the Equation 1 or 2 using one of multistep methods. Almost, they used to solve higherorder ODE by converting it to equivalent system of first-order ODEs and can solve using a classical RK method Faires and Burden (2003). However, it would be more efficient if ODEs of order five can be solved using the proposed direct RKM method. The proposed method solve Equation 1 or 2 directly be more efficient since it has less function evaluations and computational time in implementation.

For review of RK type methods, the second-order ODEs, Sommeijer (1993 Van der Houwen and Sommeijer, 1989) have derived direct numerical integrators with constant step size while Cong (2001) has derived direct numerical methods with variable step-size for solving second-order ODEs while for third-order, Mechee et al. (2013b; You and Chen, 2013) have derived direct integrators of RK type for solving ODEs of third-order while Senu et al. (2014) have derived variable step-size direct integrators of RK type of orders $6(5), 5(4)$ and 4(3) for solving third-order ODEs. Hussain et al. (2016) have derived direct numerical integrators with variable and constant step-size for solving fourth-order ODEs, moreover different orders of direct explicit RKD methods for solving special thirdorder ODEs with constant step-size have been derived (Mechee et al., 2014a; 2014b; 2014c). However, the regions of stability for RKD methods have been derived by Mechee et al. (2016).

In this study, we are concerned with the one-step RKM integrators for directly solving fifth-order ODEs. To obtain the order conditions we used the Taylor series expansion approach. Consequently, we have derived two of direct RKM integrators based on the algebraic equations of order conditions of RKM integrators.

\section{Proposed RKM Methods}

The proposed formula of explicit RKM integrator with $s$-stage for solving fifth-order ODEs (1) can be written as follow:

$$
\begin{aligned}
& w_{n+1}=w_{n}+w_{n}^{\prime}+\frac{h^{2}}{2 !} w_{n}^{\prime \prime}+\frac{h^{3}}{3 !} w_{n}^{\prime \prime \prime}+\frac{h^{4}}{4 !} w_{n}^{\prime \prime \prime \prime}+h^{5} \sum_{i=1}^{s} b_{i} k_{i} \\
& w_{n+1}^{\prime}=w_{n}^{\prime}+h w_{n}^{\prime \prime}+\frac{h^{2}}{2 !} w_{n}^{\prime \prime \prime}+\frac{h^{3}}{3 !} w_{n}^{\prime \prime \prime \prime}+h^{4} \sum_{i=1}^{s} b_{i}^{\prime} k_{i} \\
& w_{n+1}^{\prime \prime}=w_{n}^{\prime \prime \prime}+h w_{n}^{\prime \prime \prime}+\frac{h^{2}}{2 !} w_{n}^{\prime \prime \prime \prime}+h^{3} \sum_{i=1}^{s} b_{i}^{\prime \prime} k_{i} \\
& w_{n+1}^{\prime \prime \prime}=w_{n}^{\prime \prime \prime}+h w_{n}^{\prime \prime \prime \prime}+h^{2} \sum_{i=1}^{s} b_{i}^{\prime \prime \prime} k_{i} \\
& w_{n+1}^{\prime \prime \prime \prime}=w_{n}^{\prime \prime \prime \prime}+h \sum_{i=1}^{s} b_{i}^{\prime \prime \prime \prime} k_{i}
\end{aligned}
$$

where:

$k_{1}=g\left(x_{n}, w_{n}\right)$

$k_{i}=g\left(\begin{array}{l}x_{n}+c_{i} h, w_{n}+h c_{i} w_{n}^{\prime}+\frac{h^{2}}{2 !} c_{i}^{2} w_{n}^{\prime \prime} \\ +\frac{h^{3}}{3 !}+c_{i}^{3} w_{n}^{\prime \prime \prime} \frac{h^{4}}{4 !} c_{i} w_{0 n}^{\prime \prime \prime \prime}+h^{5} \sum_{i=1}^{i-1} a_{i j} k_{j}\end{array}\right)$

for $i=2,3, \ldots, s$. and $h$ is the step-size. The parameters of RKM integrator are $a_{i j}, c_{i}, b_{i}^{\prime \prime \prime}, b_{i}^{\prime \prime \prime}, b_{i}^{\prime \prime}, b_{i}^{\prime}$ and $b_{i}$ for $i=$ $1,2, \ldots, s$ and $j=1,2, \ldots, s$ are real. It is an explicit integrator if $a_{i j}=0$ for $i \leq j$ and otherwise its implicit integrator. We have expressed the coefficients RKM method in Butcher Table as follows: 


$$
\frac{c \mid A}{\mid \begin{array}{l}
b^{T} \\
b^{\prime T} \\
b^{\prime \prime T} \\
b^{\prime \prime \prime T} \\
b^{\prime \prime \prime \prime} T
\end{array}}
$$

The order conditions of RKD integrators for solving third-order ODEs have been derived by Mechee et al. (2013a; You and Chen, 2013) while the order conditions of RKFD integrators for solving fourth-order ODEs have been derived by Hussain et al. (2015). In this study, using the same technique, we have derived the algebraic equations of order conditions of RKM methods for solving special fifth-order ODEs.

\section{The Order Conditions Derivation of RKM Methods}

This approach has a special characteristic history, it deals with non-scalar problems in the hope that this generalizes correctly to high dimensions. It is similar to those which were used by Runge, Heun, Kutta, Nystrom, Huta and others to obtain methods up to order six for solving the first-order problem $y^{\prime}=f(x, y)$ Butcher and Wanner (1996; Butcher, 2008). The algebraic equations of order conditions of RKM integrators can be obtain from the direct expansion of the local truncation error. This idea is based on the derivation of algebraic equations of order conditions for RK methods introduced in (Dormand, 1996). The RKM formulae in (3-7) can be expressed as follow:

$$
\begin{aligned}
& w_{n+1}=w_{n}+h \Phi\left(x_{n}, w_{n}\right), \\
& w_{n+1}^{\prime}=w_{n}^{\prime}+h \Phi^{\prime}\left(x_{n}, w_{n}\right), \\
& w_{n+1}^{\prime \prime}=w_{n}^{\prime \prime}+h \Phi^{\prime \prime}\left(x_{n}, w_{n}\right), \\
& w_{n+1}^{\prime \prime \prime}=w_{n}^{\prime \prime \prime}+h \Phi^{\prime \prime \prime}\left(x_{n}, w_{n}\right), \\
& w_{n+1}^{\prime \prime \prime \prime}=w_{n}^{\prime \prime \prime \prime}+h \Phi^{\prime \prime \prime \prime}\left(x_{n}, w_{n}\right)
\end{aligned}
$$

where, the increment functions are defined as the following:

$$
\begin{aligned}
& \Phi\left(x_{n}, w_{n}\right)=w_{n}^{\prime}+\frac{h}{2 !} w_{n}^{\prime \prime}+\frac{h^{2}}{3 !} w_{n}^{\prime \prime \prime}+\frac{h^{3}}{4 !} w_{n}^{\prime \prime \prime \prime}+h^{4} \sum_{i=1}^{s} b_{i} k_{i}, \\
& \Phi^{\prime}\left(x_{n}, w_{n}\right)=w_{n}^{\prime \prime \prime}+\frac{h}{2 !} w_{n}^{\prime \prime \prime}+\frac{h^{2}}{3 !} w_{n}^{\prime \prime \prime \prime}+h^{3} \sum_{i=1}^{s} b_{i}^{\prime} k_{i}, \\
& \Phi^{\prime \prime}\left(x_{n}, w_{n}\right) w_{n}^{\prime \prime \prime \prime}+\frac{h}{2 !} w_{n}^{\prime \prime \prime \prime}+h^{2} \sum_{i=1}^{s} b_{i}^{\prime \prime} k_{i}, \\
& \Phi^{\prime \prime \prime}\left(x_{n}, w_{n}\right) w_{n}^{\prime \prime \prime \prime}+h \sum_{i=1}^{s} b_{i}^{\prime \prime \prime} k_{i}, \\
& \Phi^{\prime \prime \prime \prime}\left(x_{n}, w_{n}\right)=\sum_{i=1}^{s} b_{i}^{\prime \prime \prime \prime} k_{i}
\end{aligned}
$$

where, $k_{i}$ is defined in the formula (8) and (9). If D represents Taylor series increment function and the local truncation errors of the derivatives of the solution of order zero up to the fourth-order cab be obtained by substituting the exact solution $w(x)$ of ODE (1) into the RKM increment function. This gives:

$$
t_{n+1}^{(i)}=h\left(\Phi^{(i)}-\Delta^{(i)}\right)
$$

for $i=0,1, \ldots, 4$.

These expressions have given in elementary differentials terms also Taylor series increment can be expressed as follows:

$$
\begin{aligned}
& \Delta^{(0)}=w^{\prime}+\frac{h}{2 !} w^{\prime \prime}+\frac{h^{2}}{3 !} w^{(3)}+\frac{h^{3}}{4 !} w^{(4)}+O\left(h^{4}\right), \\
& \Delta^{(1)}=w^{\prime \prime}+\frac{h}{2 !} w^{(3)}+\frac{h^{2}}{3 !} w^{(4)}+O\left(h^{3}\right), \\
& \Delta^{(2)}=w^{(3)}+\frac{h}{2 !} w^{(4)}+O\left(h^{2}\right), \\
& \Delta^{(3)}=w^{(4)}+O(h), \\
& \Delta^{(4)}=O(1)
\end{aligned}
$$

Hence, for the scalar case function, the few first differentials of the function $g$ are given as follow:

$$
\begin{aligned}
& G_{1}^{(5)}=g, \\
& G_{1}^{(6)}=g_{x}+g_{w} w^{\prime}, \\
& G_{1}^{(7)}=g_{x x}+2 g_{x w} w^{\prime}+g_{w} w^{\prime \prime}+g_{w w}\left(w^{\prime}\right)^{2}, \\
& G_{1}^{(8)}=g_{x x x}+w^{\prime 3} g_{w w w}+g_{x w w}\left(2 w^{\prime}+w^{\prime 2}\right) \\
& +3 w^{\prime} w^{\prime \prime} g_{w w}+3 w^{\prime \prime} g_{x w}+3 w^{\prime} g_{x w x}+w^{\prime \prime \prime} g_{w}, \\
& G_{1}^{(9)}=g_{x x x x}+w^{\prime} g_{x x x w}+\left(w^{\prime}\right)^{3}\left(w^{\prime} g_{w w w w}+g_{w w w x}\right) \\
& +3\left(w^{\prime}\right)^{2} w^{\prime \prime} g_{w w w}+2 w^{\prime \prime} g_{x w w}\left(1+w^{\prime}\right)+w^{\prime}, \\
& \left(g_{x w w x}+y^{\prime} g_{x w w w}\left(2+w^{\prime}\right)+3 w^{\prime} w^{\prime \prime}\left(w^{\prime} g_{w w w}+g_{w w x}\right)\right. \\
& +3 g_{w w}\left(w^{\prime}+w^{\prime \prime}\left(w^{\prime \prime}+w^{\prime}\right)\right)+3 w^{\prime \prime \prime} g_{x w} \\
& \left.+3 w^{\prime \prime}\left(g_{x w x}+w^{\prime} g_{x w w}\right)+3 w^{\prime \prime} g_{x w x}+3 w^{\prime}\left(g_{x w x x}+\right) w^{\prime} g_{x w x w}\right) \\
& +w^{\prime \prime \prime \prime} g_{w}+w^{\prime \prime \prime}\left(g_{w x}+w^{\prime} g_{w w}\right)
\end{aligned}
$$

The increment functions $\Phi^{(0)}, \Phi^{(1)}, \Phi^{(2)}, \Phi^{(3)}$ and $\Phi^{(4)}$ for the RKM integrators can be written as follow using the above terms:

$$
\begin{aligned}
& \sum_{i=1}^{s} b_{i} k_{i}=\sum_{i=1}^{s} b_{i} g+\sum_{i=1}^{s} b_{i} c_{i}\left(g_{x}+g_{y} y^{\prime}\right) h+ \\
& \frac{1}{2} \sum_{i=1}^{s} b_{i} c_{i}^{2}\left(g_{x x}+2 g_{x y} y^{\prime}+g_{y} y^{\prime \prime}+g_{y y}\left(y^{\prime}\right)^{2}\right) h^{2}+O\left(h^{3}\right)
\end{aligned}
$$




$$
\begin{aligned}
& \sum_{i=1}^{s} b_{i}^{\prime} k_{i}=\sum_{i=1}^{s} b_{i}^{\prime} g+\sum_{i=1}^{s} b_{i}^{\prime} c_{i}\left(g_{x}+g_{y} y^{\prime}\right) h+ \\
& \frac{1}{2} \sum_{i=1}^{s} b_{i}^{\prime} c_{i}^{2}\left(g_{x x}+2 g_{x y} y^{\prime}+g_{y} y^{\prime \prime}+g_{y y}\left(y^{\prime}\right)^{2}\right) h^{2}+O\left(h^{3}\right) \\
& \sum_{i=1}^{s} b_{i}^{\prime \prime} k_{i}=\sum_{i=1}^{s} b_{i}^{\prime \prime} g+\sum_{i=1}^{s} b_{i}^{\prime \prime} c_{i}\left(g_{x}+g_{y} y^{\prime}\right) h+ \\
& \frac{1}{2} \sum_{i=1}^{s} b_{i}^{\prime \prime} c_{i}^{2}\left(g_{x x}+2 g_{x y} y^{\prime}+g_{y} y^{\prime \prime}+g_{y y}\left(y^{\prime}\right)^{2}\right) h^{2}+O\left(h^{3}\right) \\
& \sum_{i=1}^{s} b_{i}^{\prime \prime \prime} k_{i}=\sum_{i=1}^{s} b_{i}^{\prime \prime \prime} g+\sum_{i=1}^{s} b_{i}^{\prime \prime \prime} c_{i}\left(g_{x}+g_{y} y^{\prime}\right) h+ \\
& \frac{1}{2} \sum_{i=1}^{s} b_{i}^{\prime \prime \prime} c_{i}^{2}\left(g_{x x}+2 g_{x y} y^{\prime}+g_{y} y^{\prime \prime}+g_{y y}\left(y^{\prime}\right)^{2}\right) h^{2}+O\left(h^{3}\right) \\
& \sum_{i=1}^{s} b_{i}^{\prime \prime \prime \prime} k_{i}=\sum_{i=1}^{s} b_{i}^{\prime \prime \prime} g+\sum_{i=1}^{s} b_{i}^{\prime \prime \prime \prime} c_{i}\left(g_{x}+g_{y} y^{\prime}\right) h+ \\
& \frac{1}{2} \sum_{i=1}^{s} b_{i}^{\prime \prime \prime \prime} c_{i}^{2}\left(g_{x x}+2 g_{x y} y^{\prime}+g_{y} y^{\prime \prime}+g_{y y}\left(y^{\prime}\right)^{2}\right) h^{2}+O\left(h^{3}\right)
\end{aligned}
$$

The expressions for the local truncation errors in the derivatives of the solution up to the fourth-order, $y^{(i)}(x)$ for $i=0,1,2,3,4$ are written as follow:

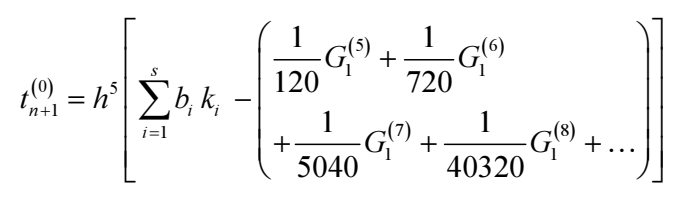

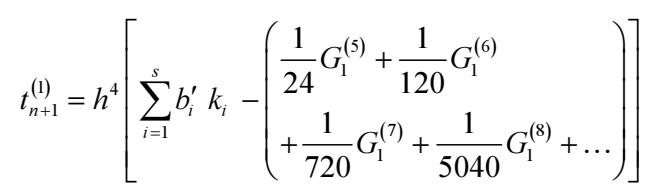

$$
\begin{aligned}
& t_{n+1}^{(2)}=h^{3}\left[\sum_{i=1}^{s} b_{i}^{\prime \prime} k_{i}-\left(\begin{array}{l}
\frac{1}{6} G_{1}^{(5)}+\frac{1}{24} G_{1}^{(6)} \\
+\frac{1}{120} G_{1}^{(7)}+\frac{1}{720} G_{1}^{(8)}+\ldots
\end{array}\right)\right]
\end{aligned}
$$

$$
\begin{aligned}
& t_{n+1}^{(3)}=h^{2}\left[\sum_{i=1}^{s} b_{i}^{\prime \prime \prime} k_{i}-\left(\begin{array}{l}
\frac{1}{2} G_{1}^{(5)}+\frac{1}{6} G_{1}^{(6)} \\
+\frac{1}{24} G_{1}^{(7)}+\frac{1}{120} G_{1}^{(8)}+\ldots
\end{array}\right)\right] \\
& t_{n+1}^{(4)}=h\left[\sum_{i=1}^{s} b_{i}^{\prime \prime \prime \prime} k_{i}-\left(\begin{array}{l}
G_{1}^{(5)}+\frac{1}{2} G_{1}^{(6)}+\frac{1}{6} G_{1}^{(7)} \\
+\frac{1}{24} G_{1}^{(8)}+\frac{1}{120} G_{1}^{(9)}+\ldots
\end{array}\right)\right]
\end{aligned}
$$

Substitute the Equations (10-14) into the Equations (15-19) respectively and expanding as a Taylor expansion. Using computer algebra which has written by MAPLE programming (Gander and Gruntz, 1999) then, the error equations or the algebraic equations of order conditions up to order-seven can be expressed in rooted trees form (Table 1-5) which have all indices are from 1 to $s$ for $w^{(i)}$ for $i=0,1, \ldots, 4$.

\section{Derivation of RKM Methods}

To derive RKM methods of orders fifth and sixth, we have used the algebraic conditions up to order of the method for $w^{(i)}$ for $i=0,1, \ldots, 4$ in Table $1-5$, respectively, with the following assumption:

$$
\begin{aligned}
& b_{i}^{\prime \prime \prime}=\left(1-c_{i}\right) b_{i}^{\prime \prime \prime \prime}, \quad b_{i}^{\prime \prime}=\frac{\left(1-c_{i}\right)^{2}}{2 !} b_{i}^{\prime \prime \prime}, \\
& b_{i}^{\prime}=\frac{\left(1-c_{i}\right)^{3}}{3 !} b_{i}^{\prime \prime \prime \prime}, \quad b_{i}=\frac{\left(1-c_{i}\right)^{4}}{4 !} b_{i}^{\prime \prime \prime \prime}
\end{aligned}
$$

\begin{tabular}{|c|c|c|c|c|}
\hline$|t|$ & $t$ & $S(t)$ & $\phi(t)$ & $x(t)$ \\
\hline 5 & $\bullet$ & HA & $\sum_{i=1}^{s} b_{i}$ & 120 \\
\hline 6 & ? & HACACA & $\sum_{i=1}^{s} b_{i} c_{i}$ & 720 \\
\hline 7 & $q$ & $\Rightarrow+\alpha^{\circ}$ & $\sum_{i=1}^{s} b_{i} c_{i}^{2}$ & 2520 \\
\hline 7 & $q$ & 0.0 .0 & $\sum_{i=1}^{s} b_{i} c_{i}^{3}$ & 840 \\
\hline
\end{tabular}

for $i=1, \ldots, s$, which imposed to reduce the number of algabraic order equations to be solve. The parameters of RKM method are $c_{i}, a_{i j}, b_{i}, b_{i}^{\prime}, b_{i}^{\prime \prime}, b_{i}^{\prime \prime \prime}, b_{i}^{\prime \prime \prime}$ for $i=1,2, \ldots, s$ and $j=1,2, \ldots, s$. The parameters of the new methods have been evaluated by solving the system of algebraic equations of order conditions in (1-5).

Three-stage fifth-order and four-stage sixth-order direct RKM integrators have been derived and the Butcher tableaus of these integrators are shown in the Table 6 and 7, respectively.

Table 1. Rooted trees of order conditions for $w$ 
Mohammed S. Mechee and Murtaza A. Kadhim / American Journal of Applied Sciences 2016, 13 (12): 1452.1460 DOI: 10.3844/ajassp.2016.1452.1460

Table 2. Rooted trees of order conditions for $w^{\prime}$

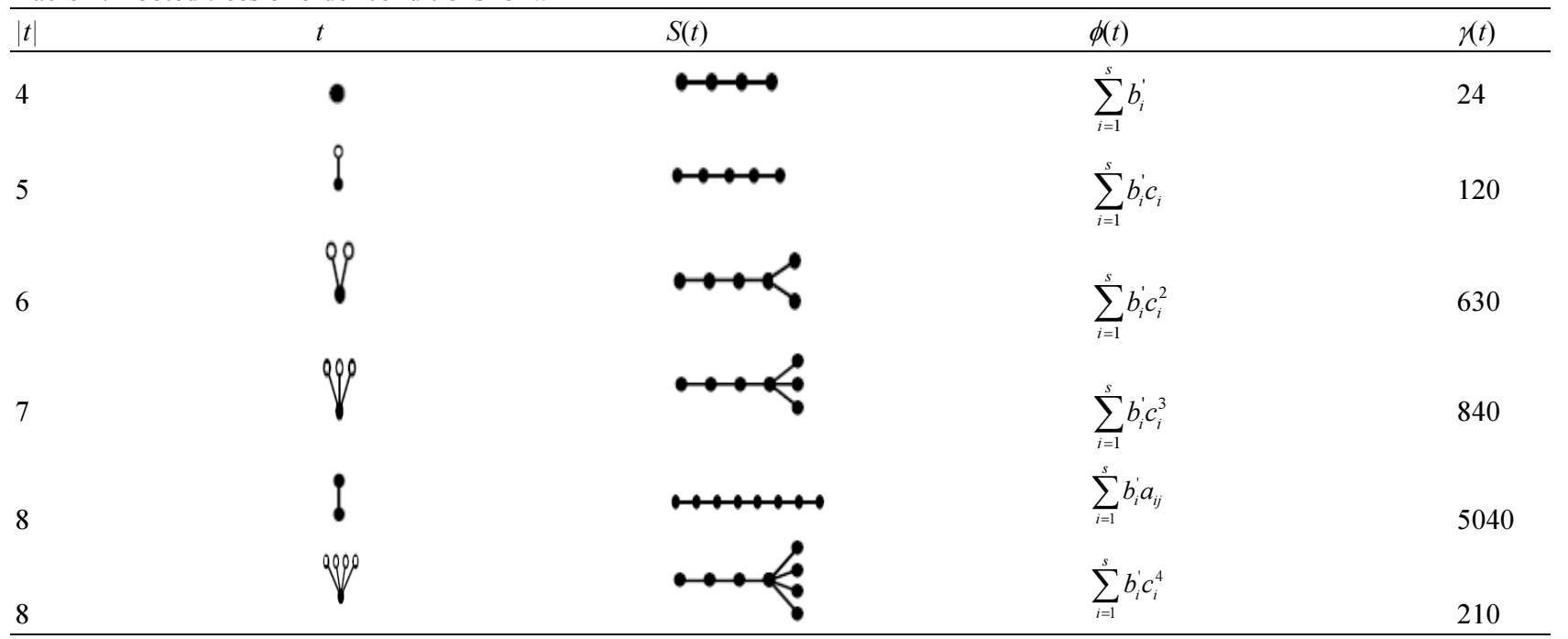

Table 3. Rooted trees of order conditions for $w^{\prime \prime}$

\begin{tabular}{|c|c|c|c|c|}
\hline$|t|$ & $t$ & $S(t)$ & $\phi(t)$ & $\chi(t)$ \\
\hline 3 & 1 & $\mapsto$ & $\sum_{i=1}^{s} b_{i}^{\prime \prime}$ & 6 \\
\hline 4 & ? & & $\sum_{i=1}^{s} b_{i}^{\prime \prime} c_{i}$ & 24 \\
\hline 5 & & & & 60 \\
\hline 6 & $\gamma$ & & $\sum_{i=1}$ & 120 \\
\hline 7 & $\bullet$ & & $\sum_{i=1}^{s} b_{i}^{\prime \prime} a_{i j}$ & 720 \\
\hline 7 & $q p$ & & $\sum_{i=1}^{s} b_{i}^{\prime \prime} c_{i}^{4}$ & 210 \\
\hline 8 & $\vartheta$ & & $\sum_{i=1}^{s} b_{i}^{\prime \prime} a_{i j} c_{j}$ & 5040 \\
\hline $\mid \overrightarrow{|t|}$ & $t$ & $S(t)$ & $\phi(t)$ & $\chi(t)$ \\
\hline 2 & 1 & $H$ & $\sum_{i=1} b$ & 2 \\
\hline 3 & ? & $H$ & $\sum_{i=1}^{s} b_{i}^{\prime \prime \prime} c_{i}$ & 6 \\
\hline 4 & & & $\sum_{i=1} b_{i}$ & 12 \\
\hline 5 & 990 & & $\sum_{i=1}^{s} b_{i}^{\prime \prime \prime} c_{i}^{3}$ & 20 \\
\hline 6 & $q 90$ & & $\sum_{i=1}^{s} b_{i}^{\prime \prime \prime} c_{i}^{4}$ & 30 \\
\hline 7 & ! & & $\sum_{i=1}^{s} b_{i}^{\prime \prime} a_{i j} c_{j}$ & 5040 \\
\hline 7 & apope & a & $\sum_{i=1}^{s} b_{i}^{\prime \prime \prime} c_{i}^{5}$ & 42 \\
\hline
\end{tabular}


Table 5. Rooted trees of order conditions for $w^{\prime \prime \prime \prime}$

\begin{tabular}{|c|c|c|c|c|}
\hline$|t|$ & $t$ & $S(t)$ & $\phi(t)$ & $\not(t)$ \\
\hline 1 & ' & ' & $\sum_{i=1}^{s} b_{i}^{\prime \prime \prime \prime}$ & 1 \\
\hline 2 & 9 & $\omega$ & $\sum_{i=1}^{s} b_{i}^{\prime \prime \prime} c_{i}$ & 2 \\
\hline 3 & $q$ & $\vdots$ & $\sum_{i=1}^{s} b_{i}^{\prime \prime \prime \prime} c_{i}^{2}$ & 3 \\
\hline 4 & 99 & \& & $\sum_{i=1}^{s} b_{i}^{\prime \prime \prime \prime} c_{i}^{3}$ & 4 \\
\hline 5 & $i$ & 1 & $\sum_{i=1}^{s} b_{i}^{\prime \prime \prime \prime} c_{i}^{4}$ & 5 \\
\hline 6 & qQp & 孚 & $\sum_{i=1}^{s} b_{i}^{\prime \prime \prime \prime} c_{i}^{5}$ & 6 \\
\hline 6 & 1 & Hat & $\sum_{i=1}^{s} b_{i}^{\prime \prime \prime \prime} a_{i j}$ & 720 \\
\hline 7 & 1 & HWH & $\sum_{i=1}^{s} b_{i}^{\prime \prime \prime} a_{i j} c_{j}$ & 5040 \\
\hline 7 & 9 & $<\ldots$ & $\sum_{i=1}^{s} b_{i}^{\prime \prime \prime} c_{i} a_{i j}$ & 840 \\
\hline 7 & $q$ & $\Leftrightarrow$ & $\sum_{i=1}^{s} b_{i}^{\prime \prime \prime \prime} c_{i}^{6}$ & 7 \\
\hline
\end{tabular}

Table 6. The butcher tableau RKM5 Method

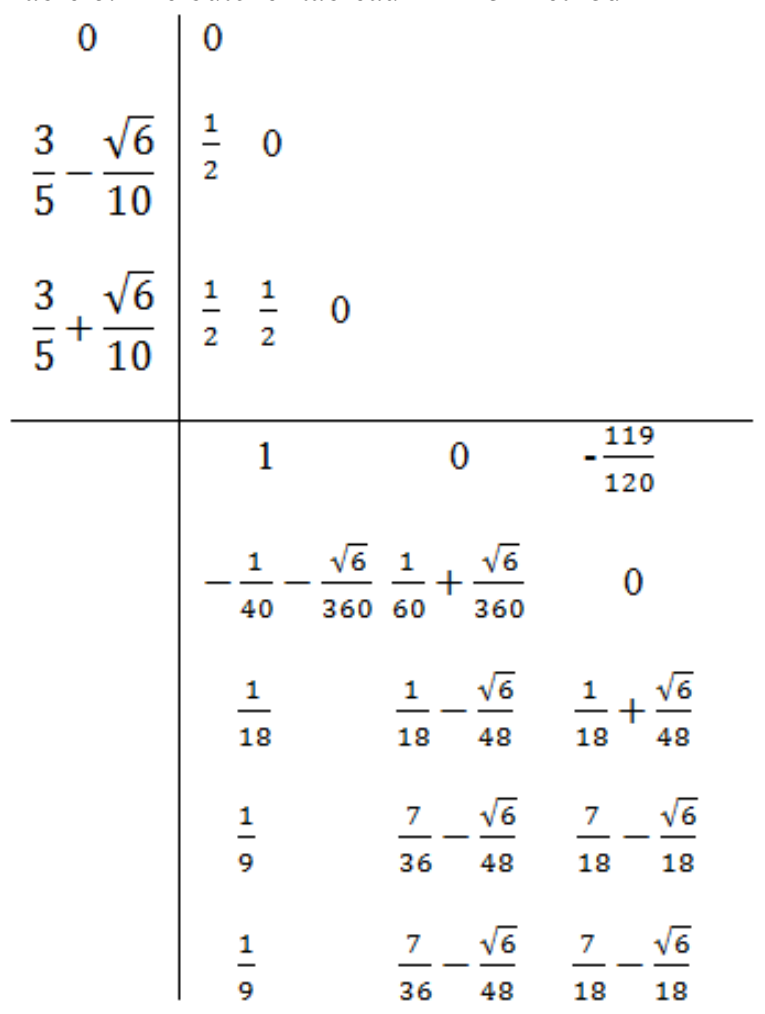

Table 7. The butcher tableau RKM6 Method

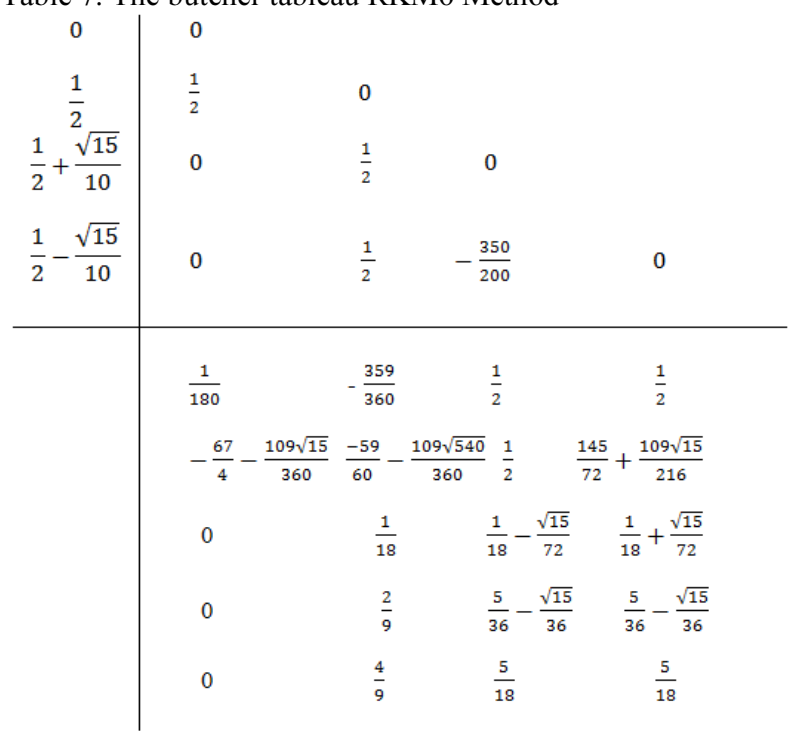

\section{Numerical Implementation}

In this section, a set of fifth-order ODEs is solved by using the fifth and sixth -order RKM methods. Then, these problems of fifth-order ODEs are reduced to a first-order ODEs systems and solved by using existing RK integrators of the same order, five and six 
respectively. The numerical results are compared in Figure 2-4 to indicate the log of maximum absolute errors against the $\log$ of total time. The notations that were used are as follows:

- $\quad$ Step: Stepsize used

- IC: Initial condition

- Ex: Exact solution

- RKM5: The direct RKM method of fifth-order

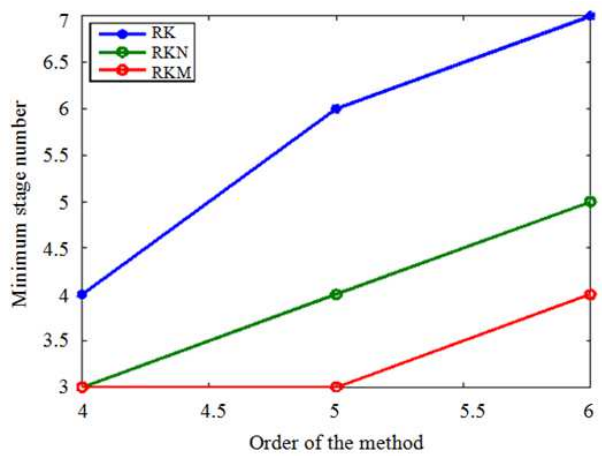

(a)
- RKM6: The direct RKM method of sixth-order

- RK5: Existing RK method of fifth-order

- RK6: Existing RK method of sixth-order as given by Dormand (1996)

- Total Time/Time: The total time in seconds to solve the problems

- Max Error: Maximum $\left|y_{(x n)}-y_{n}\right|$ is maximum of absolute errors of the true solutions and the computed solutions

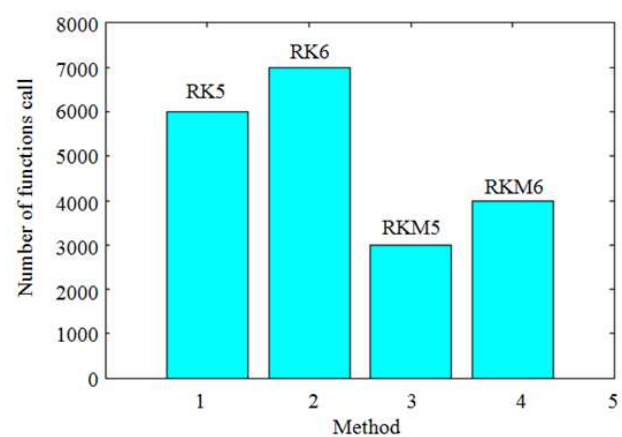

(b)

Fig. 1. (a) Minimum stage number versus method order for RK, RKN and the recently proposed RKM method, (b) Number of function calls for RK and RKM methods with orders 5,6

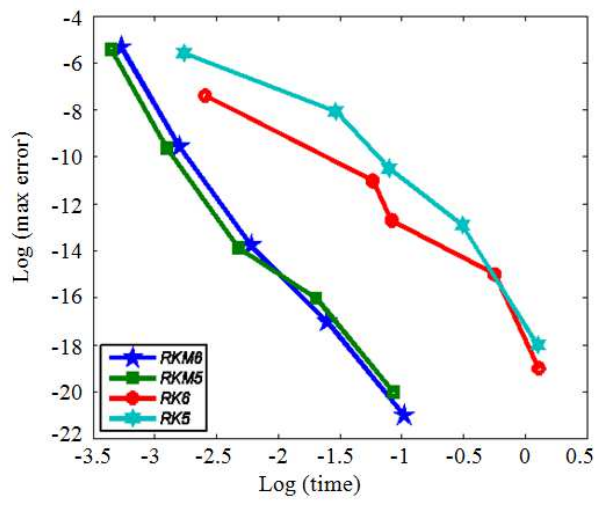

(a)

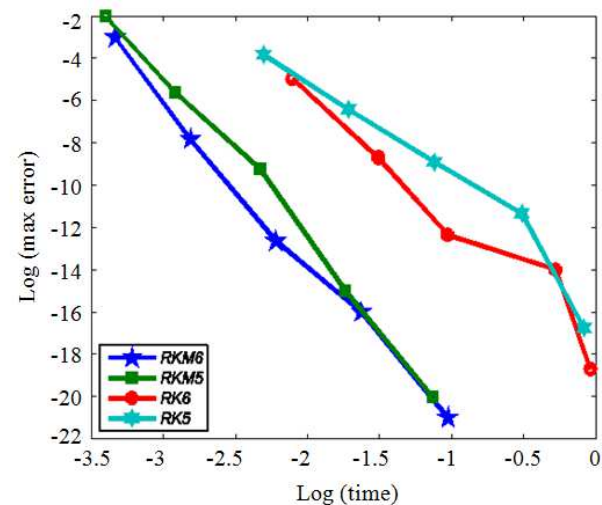

(b)

Fig. 2. Errors versus computational time for RKM5, RKM6, RK5 and RK6 methods in problems (a) 1 and (b) 2

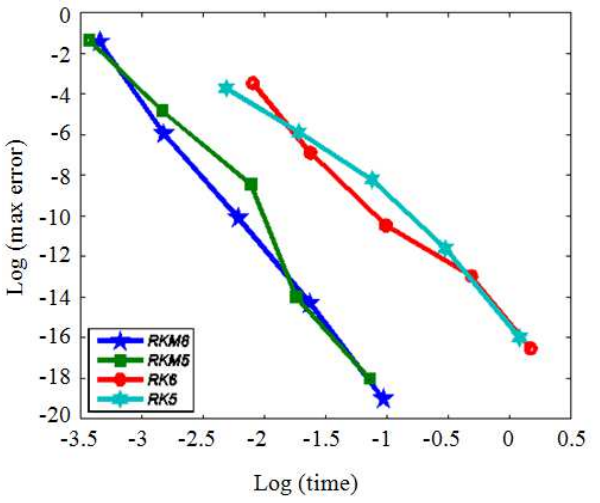

(a)

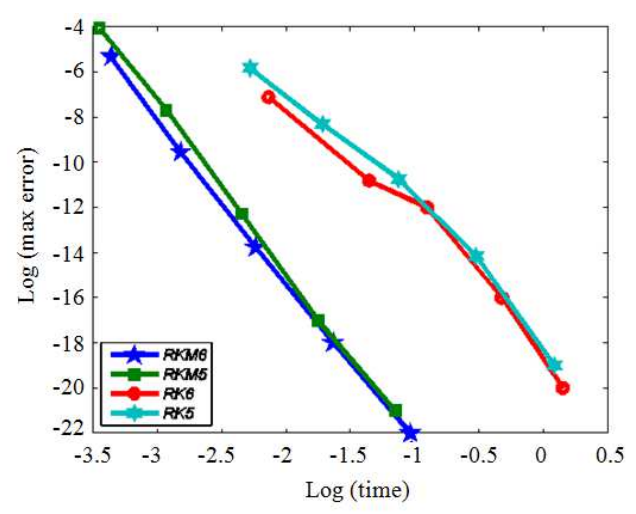

(b)

Fig. 3. Errors versus computational time for RKM5, RKM6, RK5 and RK6 methods in problems (a) 3 and (b) 4 


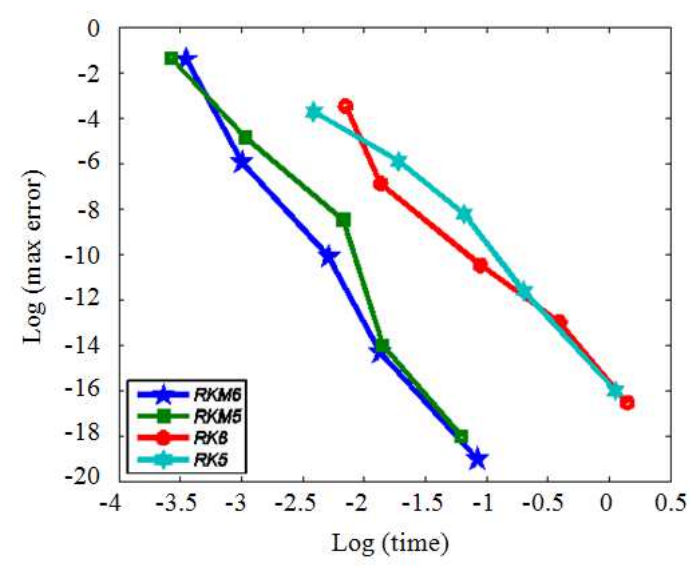

(a)

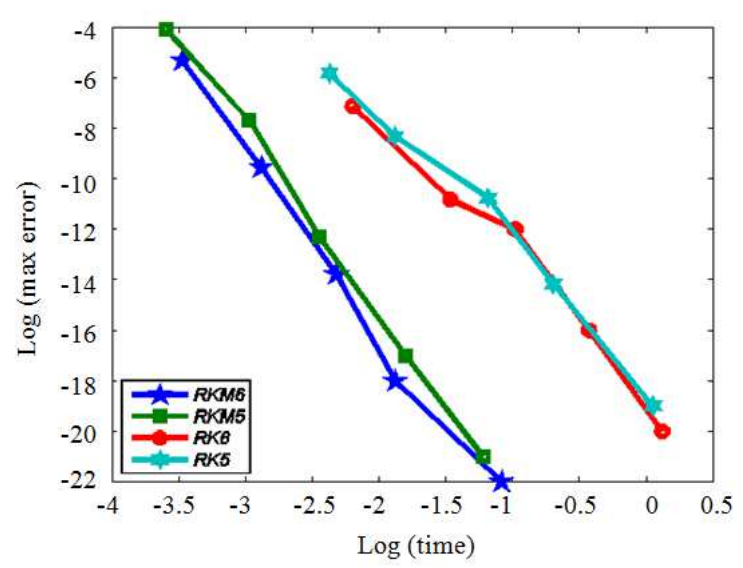

(b)

Fig. 4. Errors versus computational time for RKM5, RKM6, RK5 and RK6 methods in problems (a) 5 and (b) 6

\section{Problems Tested of ODEs}

Problem 1 (Linear ODE)

$$
y^{(5)}(t)=\cos (t) ; \quad 0<t \leq \pi
$$

- $\quad$ IC: $y^{(2 i+1)}(0)=(-1)^{i}, y^{(2 i)}(0)=0$, for $i=0,1$ and $y^{(4)}(0)=0$

- $\operatorname{Ex}: y(t)=\sin (t)$

Problem 2 (Non Constant Coefficients ODE)

$$
y^{(5)}(t)=\left(-32 t^{5}+16 t^{4}-8 t^{3}+4 t^{2}-2 t\right) y(t), \quad 0<t \leq 1
$$

- IC: $y(0)=1, y^{(i)}(0)=0$, for $i=1,2,3,4$

- $\quad$ Ex: $y(t)=e^{-12}$

\section{Problem 3 (Non Linear ODE)}

$$
y^{(5)}(t)=-120 y^{6}(t), \quad 0<t \leq 1
$$

- $\quad$ IC: $y^{(i)}(0)=(-1)^{i} i$ ! for $i=0,1, \ldots, 4$

- $\operatorname{Ex}: y(t)=\frac{1}{1+t}$

\section{Problem 4 (Linear ODE)}

$$
y^{(5)}(t)=-y(t), \quad 0<t \leq 1
$$

- $\quad \mathrm{IC}: y^{(i)}(0)=(-1)^{i}$ for $i=0,1, \ldots, 4$

- $\quad$ Ex: $y(t)=e^{-t}$

Problem 5 (Linear ODE with Relatively Long Interval)

$$
y^{(5)}(t)=0.00001 y(t), \quad 0<t \leq 10
$$

- $\quad$ IC: $y^{(i)}(0)=(-0.1)^{i}$ for $i=0,1, \ldots, 4$

- Ex: $y(t)=e^{-\frac{1}{10}}$

Problem 6 (Linear System of ODEs)

$$
\begin{aligned}
& y_{1}^{(5)}(t)=-212 y_{1}(t)-180 y_{2}(t)-211 y_{3}(t), \\
& y_{2}^{(5)}(t)=-212 y_{1}(t)-179 y_{2}(t)-211 y_{3}(t), \\
& y_{3}^{(5)}(t)=-242 y_{1}(t)-242 y_{2}(t)-243 y_{3}(t)
\end{aligned}
$$

IC:

$$
\begin{aligned}
& y_{1}(0)=1, y_{1}^{\prime}(0)=-2, y_{1}^{\prime \prime}(0)=6, y_{1}^{\prime \prime \prime}(0)=-20, y_{1}^{(4)}(0)=66 \\
& y_{2}(0)=0, y_{2}^{\prime}(0)=1, y_{2}^{\prime \prime}(0)=-5, y_{2}^{\prime \prime \prime}(0)=19, y_{2}^{(4)}(0)=65 \\
& y_{3}(0)=0, y_{3}^{\prime}(0)=-2, y_{3}^{\prime \prime}(0)=8, y_{3}^{\prime \prime \prime}(0)=-26, y_{3}^{(4)}(0)=80
\end{aligned}
$$

The system is integrated over the interval $[0,2]$.

Ex:

$$
\begin{aligned}
& y_{1}(t)=e^{-t}-e^{-2 t}+e^{-3 t}, \\
& y_{2}(t)=e^{-2 t}-e^{-3 t}, \\
& y_{3}(t)=e^{-3 t}-e^{-t}
\end{aligned}
$$

\section{Discussion and Conclusion}

In this study, we have derived the algebraic equations of order conditions for direct integrators of RKM for special fifth-order ordinary differential equations. Our approach is based on Taylor series expansion. We have derived two RKM methods of three-stage fifth-order, four-stage sixth-order respectively. Numerical results of the implementation show that the new integrators are as accurate as well-known existing methods; however, they are more efficient in implementation as they require less function evaluations. As such, we conclude that these 
integrators are costly effective, in terms of computation time and number of evaluation than existing methods.

\section{Acknowledgement}

The authors would like to thank university of Kufa for supporting this research project.

\section{Author's Contributions:}

Mohammed S. Mechee: Analysis of the method, numerical simulation and writing.

Murtaza A. Kadhim: Analysis of the method, discussions and writing.

\section{Ethics}

There are no ethical issues associated with this work.

\section{References}

Butcher, J. and G. Wanner, 1996. Runge-Kutta methods: Some historical notes. Applied Numerical Math., 22: 113-151. DOI: 10.1016/S0168-9274(96)00048-7

Butcher, J.C., 2008. Numerical Methods for Ordinary Differential Equations. 2nd Edn., John Wiley \& Sons, Chichester, ISBN-10: 0470753757, pp: 482.

Cong, N.H., 2001. Explicit pseudo two-step RKN methods with stepsize control. Applied Numerical Math., 38: 135-144. DOI: 10.1016/S0168-9274(01)00031-9

Dormand, J.R., 1996. Numerical Methods for Differential Equations: A Computational Approach. 1st Edn., CRC Press, ISBN-10: 0849394333, pp: 384.

Faires, J.D. and R. Burden, 2003. Numerical Methods. 1st Edn., Courier Corporation, Mineola, ISBN-10: 0486428079, pp: 573.

Gander, W. and D. Gruntz, 1999. Derivation of numerical methods using computer algebra. SIAM Rev., 41: 577-593. DOI: $10.1137 / \mathrm{S} 003614459935093 \mathrm{X}$

Hussain, K., F. Ismail and N. Senu, 2015. Runge-Kutta type methods for directly solving special fourthorder ordinary differential equations. Math. Prob. Eng. DOI: 10.1155/2015/893763

Hussain, K.A., F. Ismail and N. Senu, 2016. Direct numerical method for solving a class of fourth-order partial differential equation. Global J. Pure Applied Math., 12: 1257-1272.

Isaza, P., F. Linares and G. Ponce, 2015. Decay properties for solutions of fifth order nonlinear dispersive equations. J. Different. Equ., 258: 764-795. DOI: $10.1016 /$ j.jde.2014.10.004
Lee, C., 2014. Some remarks on the fifth-order KdV equations. J. Math. Analysis Applic., 425: 281-294. DOI: $10.1016 /$ j.jmaa.2014.10.021

Mechee, M.S., F. Ismail, Z. Siri and N. Senu, 2013a. Directly solving special second order delay differential equations using runge-kutta-nyström method. Math. Prob. Eng. DOI: 10.1155/2013/830317

Mechee, M.S., F. Ismail, Z. Siri and N. Senu, 2013b. A three-stage fifth-order runge-kutta method for directly solving special third-order differential equation with application to thin film flow problem. Math. Prob. Eng. DOI: 10.1155/2013/795397

Mechee, M.S., F. Ismail, Z. Siri and N. Senu, 2014a. Direct numerical methods for solving a class of third-order partial differential equations. Applied Math. Comput., 247: 663-674. DOI: $10.1016 /$ j.amc.2014.09.021

Mechee, M.S., F. Ismail, Z. Siri and N. Senu, 2014b. A third-order direct integrators of runge-kutta type for special third-order ordinary and delay differential equations. J. Applied Sci., 7: 102-116. DOI: 10.3923/ajaps.2014.102.116

Mechee, M.S., F. Ismail, Z. Siri and N. Senu, 2014c. A four-stage sixth-order RKD method for directly solving special third-order ordinary differential equations. Life Sci. J., 11: 399-404.

Mechee, M.S., Z.M. Hussain and H.R. Mohammed, 2016. On the reliability and stability of direct explicit Runge-Kutta integrators. Global J. Pure Applied Math., 12: 3959-3975.

Senu, N., M. Mechee, F. Ismail and Z. Siri, 2014, Embedded explicit Runge-Kutta type methods for directly solving special third order differential equations $y^{\prime \prime \prime}=f(x, y)$. Applied Math. Comput., 240: 281-293. DOI: 10.1016/j.amc.2014.04.094

Sommeijer, B.P., 1993. Explicit, high-order RungeKutta-Nyström methods for parallel computers. Applied Numerical Math., 13: 221-240. DOI: $10.1016 / 0168-9274(93) 90145-\mathrm{H}$

Van der Houwen, P. and B. Sommeijer, 1989. Diagonally implicit Runge-Kutta-Nyström methods for oscillatory problems. SIAM J. Numerical Analysis, 26: 414-429. DOI: 10.1137/0726023

Wazwaz, A.M., 2006. Solitons and periodic solutions for the fifth-order $\mathrm{KdV}$ equation. Applied Math. Lett., 19: 1162-1167. DOI: 10.1016/j.aml.2005.07.014

You, X. and Z. Chen, 2013. Direct integrators of RungeKutta type for special third-order ordinary differential equations. Applied Numerical Math., 74: 128-150. DOI: 10.1016/j.apnum.2013.07.005 